\title{
A Guide for the R Package rhythm_metrics
}

\author{
Cong Zhang, Radboud University
}

\section{Contents}

1 List of Functions $\quad 2$

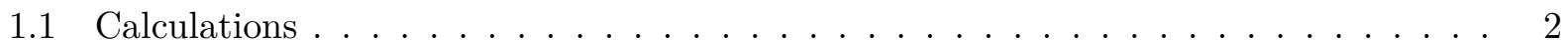

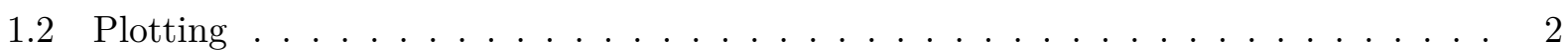

2 Installation $\quad 2$

3 Import packages $\quad 2$

4 Examples $\quad 3$

4.1 Create dataframe . . . . . . . . . . . . . . . . . . . 3

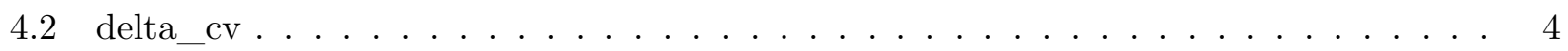

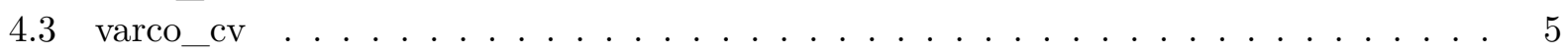

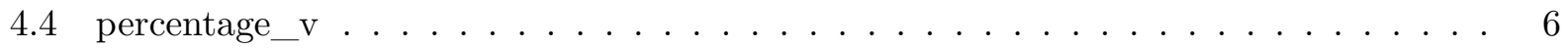

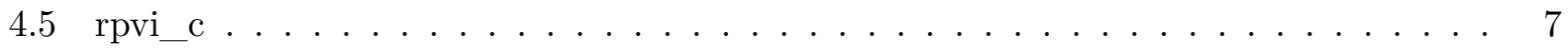

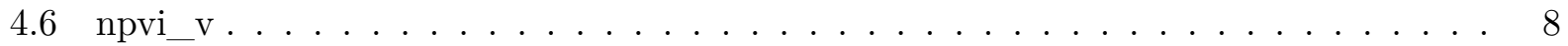




\section{List of Functions}

\subsection{Calculations}

- delta_cv

- varco_cv

- percentage_v

- rpvi_c

- npvi_v

\subsection{Plotting}

- plot_delta_cv

- plot_varco_cv

- plot_percentage_v

- plot_rpvi

- plot_npvi

\section{Installation}

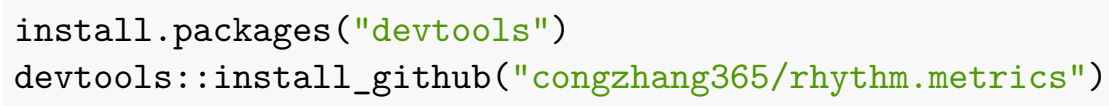

\section{Import packages}

library (rhythm.metrics)

library (dplyr) 


\section{Examples}

\subsection{Create dataframe}

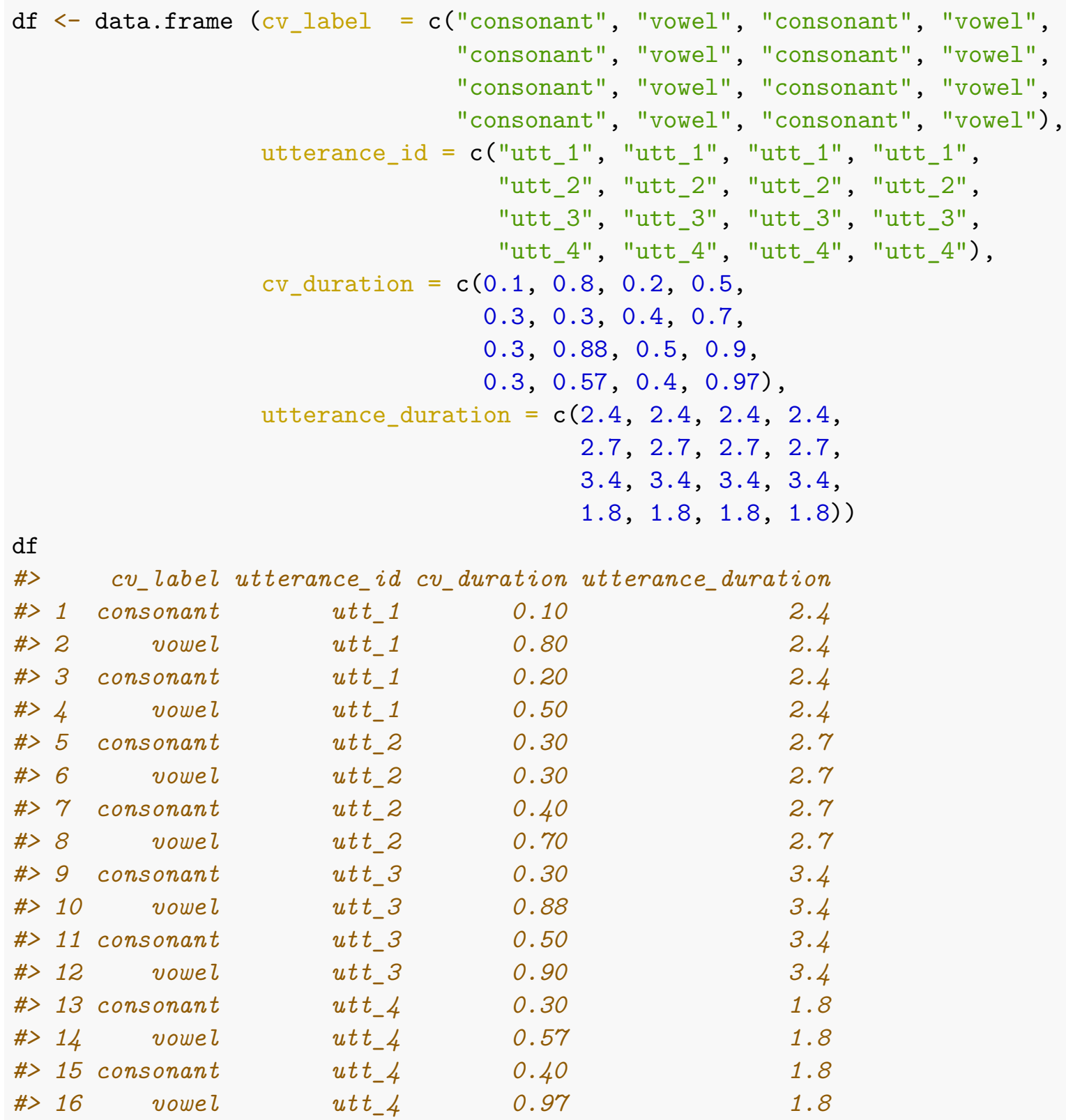




\section{2 delta_cv}

Delta C and Delta V are rhythm metrics based on Ramus, F., Nespor, M., \& Mehler, J. (1999). Correlates of linguistic rhythm in the speech signal. Cognition, 73(3), 265-292.

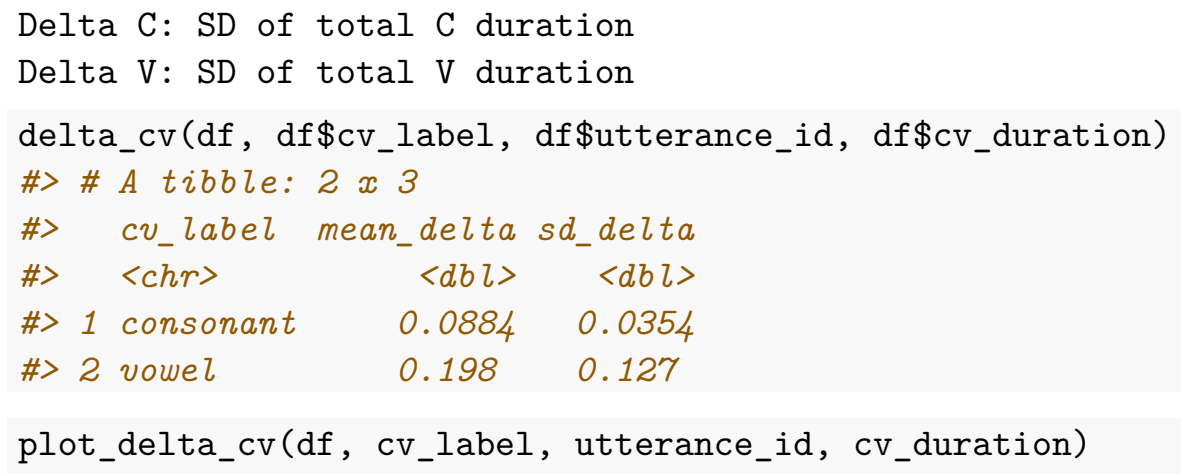




\section{3 varco_cv}

Varco C and Varco V are rhythm metrics based on Dellwo, Volker (2006). Rhythm and Speech Rate: A Variation Coefficient for deltaC. In: Karnowski, P; Szigeti, I. Language and language-processing. Frankfurt/Main: Peter Lang, 231-241.

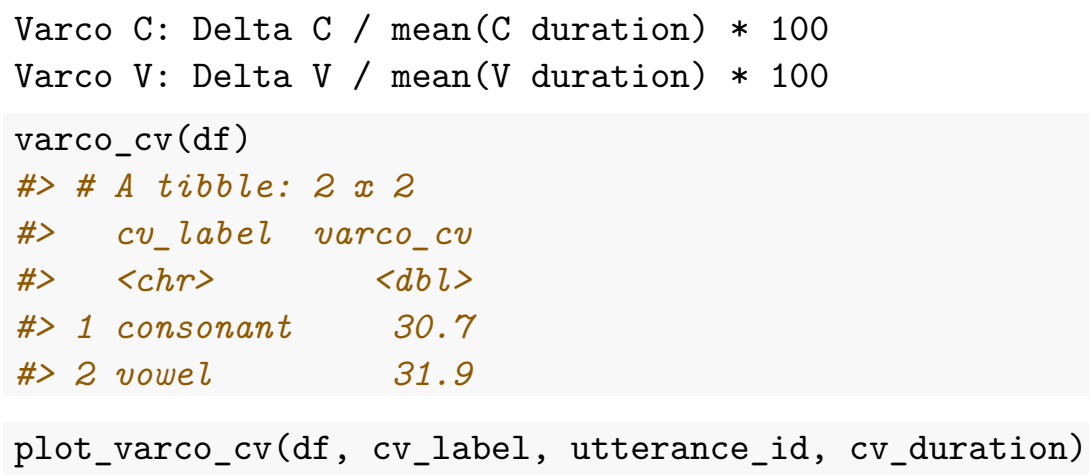




\section{4 percentage_v}

$\% \mathrm{~V}$ is a rhythm metrics based on Ramus, F., Nespor, M., \& Mehler, J. (1999). Correlates of linguistic rhythm in the speech signal. Cognition, 73(3), 265-292. It calculates the ratio of vocalic material to the total duration of an utterance.

$\% \mathrm{~V}$ : total V duration / total utterance duration






\section{5 rpvi_c}

rPVI C is a rhythm metrics based on Grabe, E., \& Low, E. L. (2002). Durational variability in speech and the rhythm class hypothesis. In Laboratory phonology 7 (pp. 515-546). De Gruyter Mouton.

It calculates the sum of the absolute differences between pairs of consecutive consonantal intervals divided by the number of pairs in the speech sample.

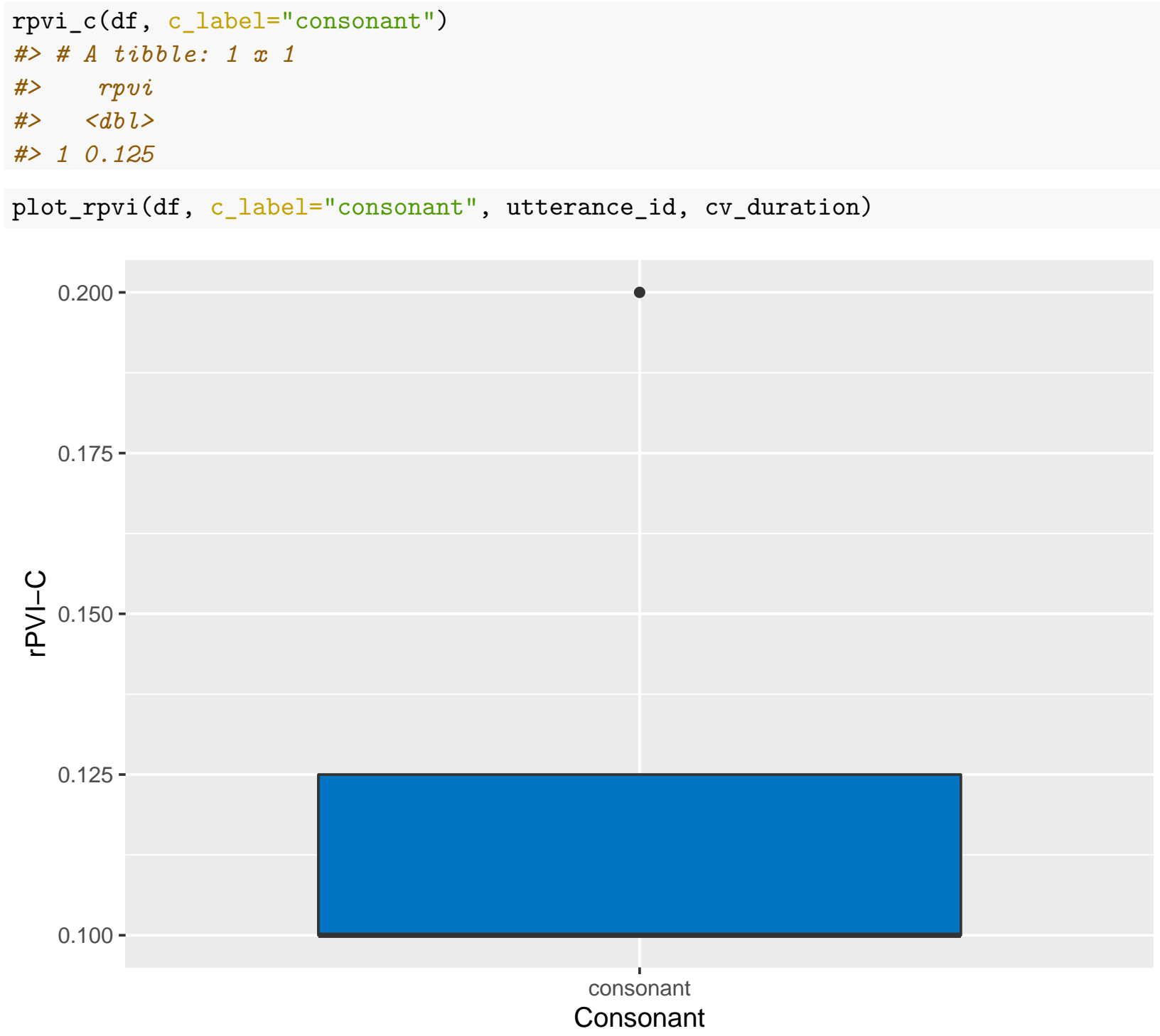




\section{6 npvi_v}

nPVI V is a rhythm metrics based on Grabe, E., \& Low, E. L. (2002). Durational variability in speech and the rhythm class hypothesis. In Laboratory phonology 7 (pp. 515-546). De Gruyter Mouton.

It calculates the normalised sum of the absolute differences between pairs of consecutive vocalic intervals divided by the number of pairs in the speech sample.
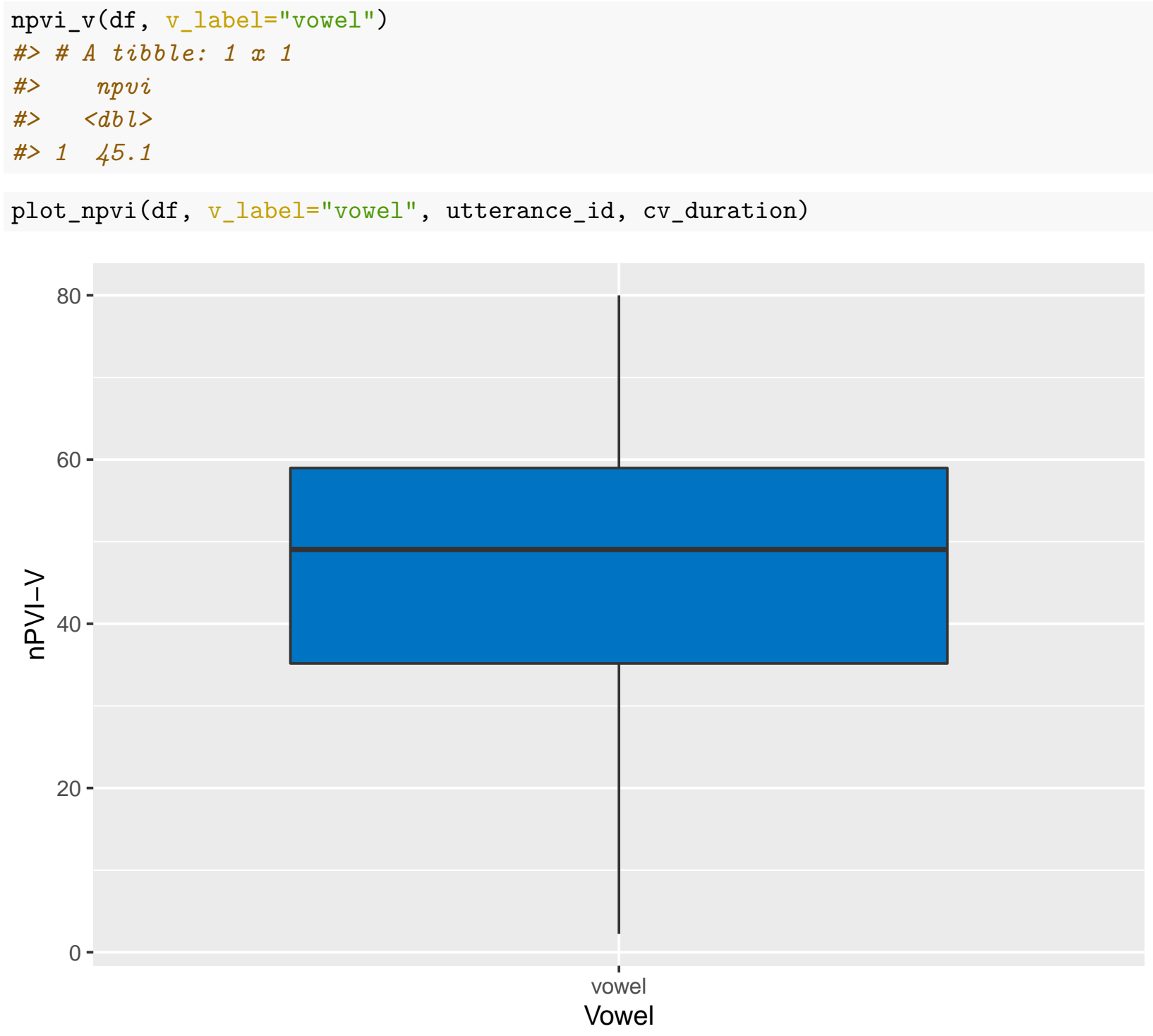